\title{
Morphometric Evaluation of Brain Infarcts in Rats and Gerbils
}

\author{
Edward F. Lundy, Brian S. Solik, Richard S. Frank, Priti S. Lacy, \\ David J. Combs, Gerald B. Zelenock, and louis G. D'Alecy
}

The Levine rat prepartion, the gerbil stroke model, and appropriate control animals were used to determine if the 2,3,5-triphenyltetrazolium chloride (TTC) would selectively identify noninfarcted versus infarcted cerebral tissue. The TTC is frequently used to quantify infarcted myocardial tissue and has been shown to have great specificity, reproducibility, and efficacy. The TTC produces a red product upon reaction with the respiratory enzymes (dehydrogenases) present in noninfarcted tissues. Irreversibly damaged tissues, lacking dehydrogenases, do not form red reaction products. Six gerbil brains and seven rat brains were incubated with the TTC, and the unreacted areas were macroscopically identified. The brains were fixed and sectioned for routine hematoxylin and eosin staining to determine the specificity of the $\Pi T C$. The $T T C$ was found to react selectively only with noninfarcted cerebral tissue. The gross brain sections were evaluated by macroscopic morphometric analysis, and the unreacted area was always ipsilateral to ligation and correlated with histologic identification of infarct. The brains from neurologically intact animals demonstrated neither macroscopic nor histological evidence of infarction. This technique allows macroscopic quantification of infarct size by planimetry. The average area of infarct for the neurologically impaired rats was $34.7 \%$ and it was $31.4 \%$ for the impaired gerbils. The percentage of surface area of each infarcted slice was found to correlate with the severity of the neurologic deficit. We conclude that the TTC staining is effective for macroscopically delineating cerebral infarcts in rats and gerbils, thus permitting quantification of infarct size.

Key Words: Brain damage; Infarct sizing; Stroke

\section{INTRODUCTION}

Contemporary cerebrovascular research utilizes various biochemical, functional, and histological end points to determine neuronal damage and the efficacy of cerebral protective interventions. However, such efforts have been hampered by the inability to quantify irreversibly damaged brain tissue objectively and accurately. The ability to macroscopically identify and quantify infarcled myocardium with 2,3,5triphenyltetrazolium chloride (TC) was a major advancement in myocardial pro-

From the Departments of Physiology, Surgery, Anatomy, and Cell Biology, The University of Michigan, Ann Arbor, Michigan.

Address reprint requests to: Dr. Louis G. D'Alecy, Department of Physiology, The University of Michigan, 7799 Medical Science Building II, Ann Arbor, MI 48109.

Received November 1, 1985; revised and accepted February 25, 1986. 
tection research. In the myocardium, this technique has been shown to have great specificity, reproducibility, and efficacy (Boor and Reynolds, 1977; Cox et al., 1968; Fishbein et al., 1981; Jennings and Kaltenbach, 1957; Jestadt and Sandritter, 1959; Laga and Munro, 1972; Lie et al., 1975; Nachlas and Shnitka, 1963; Sandritter and Jestadt, 1958; Wachstein and Meisel, 1955). The objective of this study was to evaluate the effectiveness of the TTC techniques in identifying and quantifying cerebral infarcts at a macroscopic level, rather than at the neuronal or microscopic level.

The TTC incubation and infarct sizing were performed on the brains of rats and gerbils. A unilateral infarct was produced in these animals by unilateral carotid ligation (gerbils) or unilateral carotid ligation and hypoxic exposure (Levine rats). These experimental models have been used extensively in our laboratory and by others to produce unilateral hemispheric injury (Harrison et al., 1973; Kahn, 1972; Levine, 1960; Levine and Sohn, 1969; Lundy et al., 1984, 1985; McGraw, 1977; McGraw et al., 1976; Payan and Conrad, 1977). In this study the selectivity and accuracy of the TTC reaction product was confirmed by standard histological determination of infarct at 24 and $48 \mathrm{hr}$ following the insult.

\section{MATERIALS AND METHODS}

\section{Levine Preparation and Protocol}

Seven adult, male Sprague-Dawley rats were prepared as previously described for the production of a lateralized neurological deficit (Lundy et al., 1984, 1985). In brief, halothane (2\%) anesthesia (chamber induction, mask maintenance) was used for the instrumentation. Right carotid arterial and right jugular venous cannulations were accomplished using saline-flushed polyethylene tubing (PE 50). The catheters were inserted $2-2.5 \mathrm{~cm}$, secured to the cervical musculature, and passed through a subcutaneous tunnel to the nape of the neck. The incisions were closed with would clips, and a 2-hr recovery period was allowed prior to hypoxic exposure.

Systemic hypoxia was induced within a cylindrical clear plastic chamber $30 \mathrm{~cm}$ long and $10 \mathrm{~cm}$ in diameter with large rubber stoppers at each end. The chamber was connected by polyethylene tubing to $20.9 \% \mathrm{O}_{2}$ (room air) or $4.5 \% \mathrm{O}_{2}$ premixed gas; it had two exhaust ports in the posterior stopper through which vascular cannulae and the rat tail were passed. The latter was secured with tape. The chamber interior was continuously monitored using a oxygen/temperature analyzer (YSI 2600). The chamber temperature was maintained at $30^{\circ} \mathrm{C}$ with a heat lamp. A probe was used to monitor rectal temperature.

The vascular cannulae were connected to appropriate transducers and to a multichannel oscillograph (Crass Model 70 Polygraph) to produce a continuous recording of mean and pulsatile blood pressure, heart rate, and respiratory rate.

After the 2-hr recovery period the animals were exposed to $4.5 \% \mathrm{O}_{2}$ until their mean blood pressure fell to $65 \mathrm{~mm} \mathrm{Hg}$, at which time they were removed from the chamber. Neurological examinations were performed in a blind study by trained observers at 24 or $48 \mathrm{hr}$ by using an examination developed in our laboratory to identify unilateral cerebral deficits. The six-part examination (Lundy et al., 1985) stressed unilateral motor differences by scoring posture, left hemiparesis, shuffle, 
circling, activity, and performance on a vertical screen. Performance was rated on a scale from one (normal) to five (extremely impaired).

\section{Gerbil Preparation and Protocol}

Six gerbils were anesthetized with $3 \%$ halothane anesthesia (chamber induction, mask maintenance). Through a midline cervical incision, the left common carotid artery was isolated, double ligated, and transected. Core body temperature (rectal) was maintained at $37 \pm 0.3^{\circ} \mathrm{C}$ (YSI 2600 monitor and heat lamp). The skin was closed with stainless-steel wound clips. The gerbils were neurologically evaluated at 6 and $24 \mathrm{hr}$ following ligation using a previously published neurological exam for gerbils (McGraw et al., 1976). Following the 24-hr exam, the gerbils were sacrificed. Sham operations for controls were performed for the rat and gerbil, during which the carotid vessels were identified but not ligated.

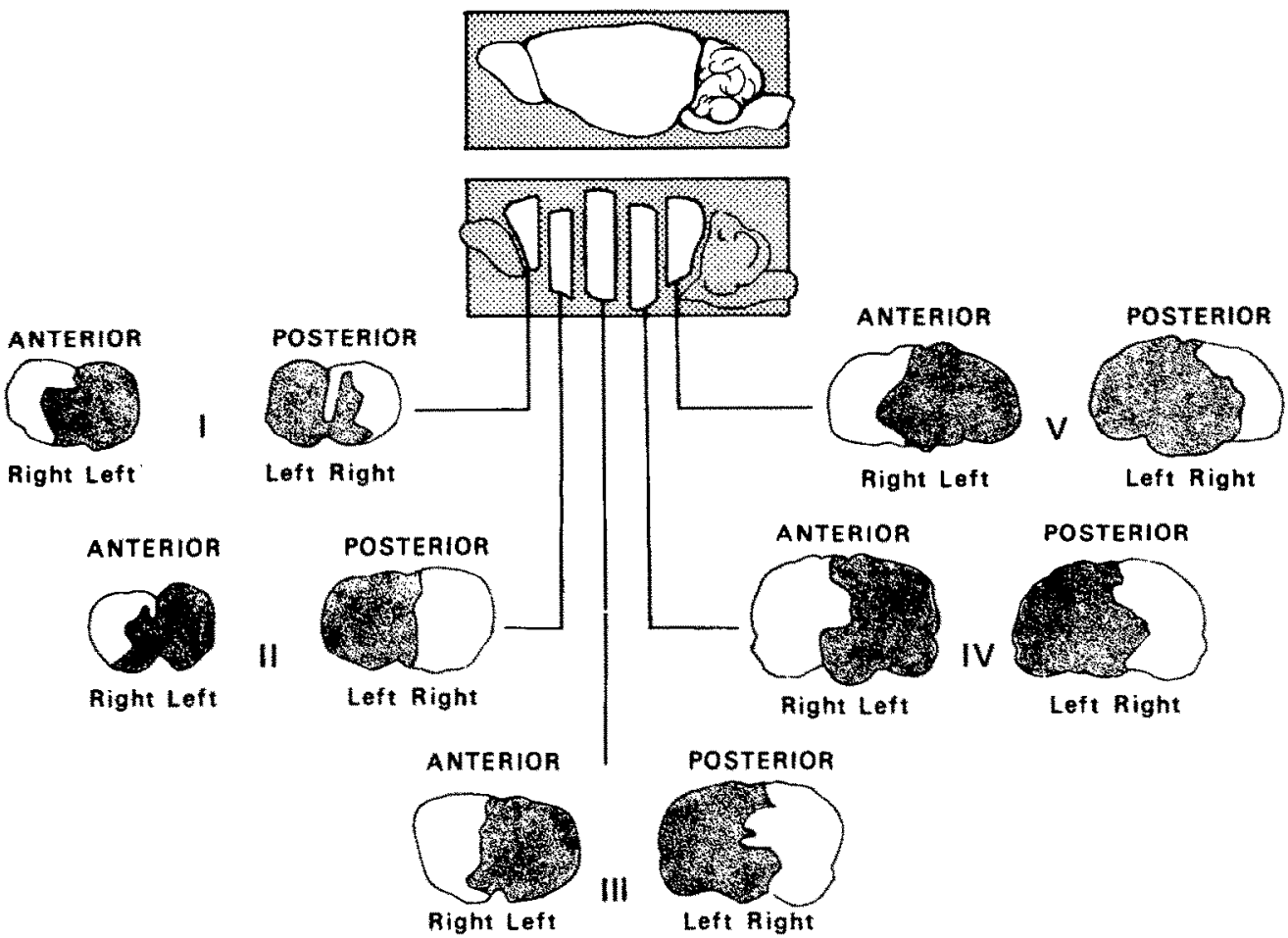

FIGURE 1. The rat and gerbil brains were cut into five slices prior to reaction with $\mathrm{TrC}$. The olfactory bulb, cerebellum, and brain stem were removed prior to incubation. Tracings were made of the anterior and posterior faces of the five brain slices (I-V). The white regions are areas of no reaction (infarcted) and the stippled areas represent areas of normal reaction (not infarcted) of a damaged rat brain after $\Pi \mathrm{CC}$ incubation. 


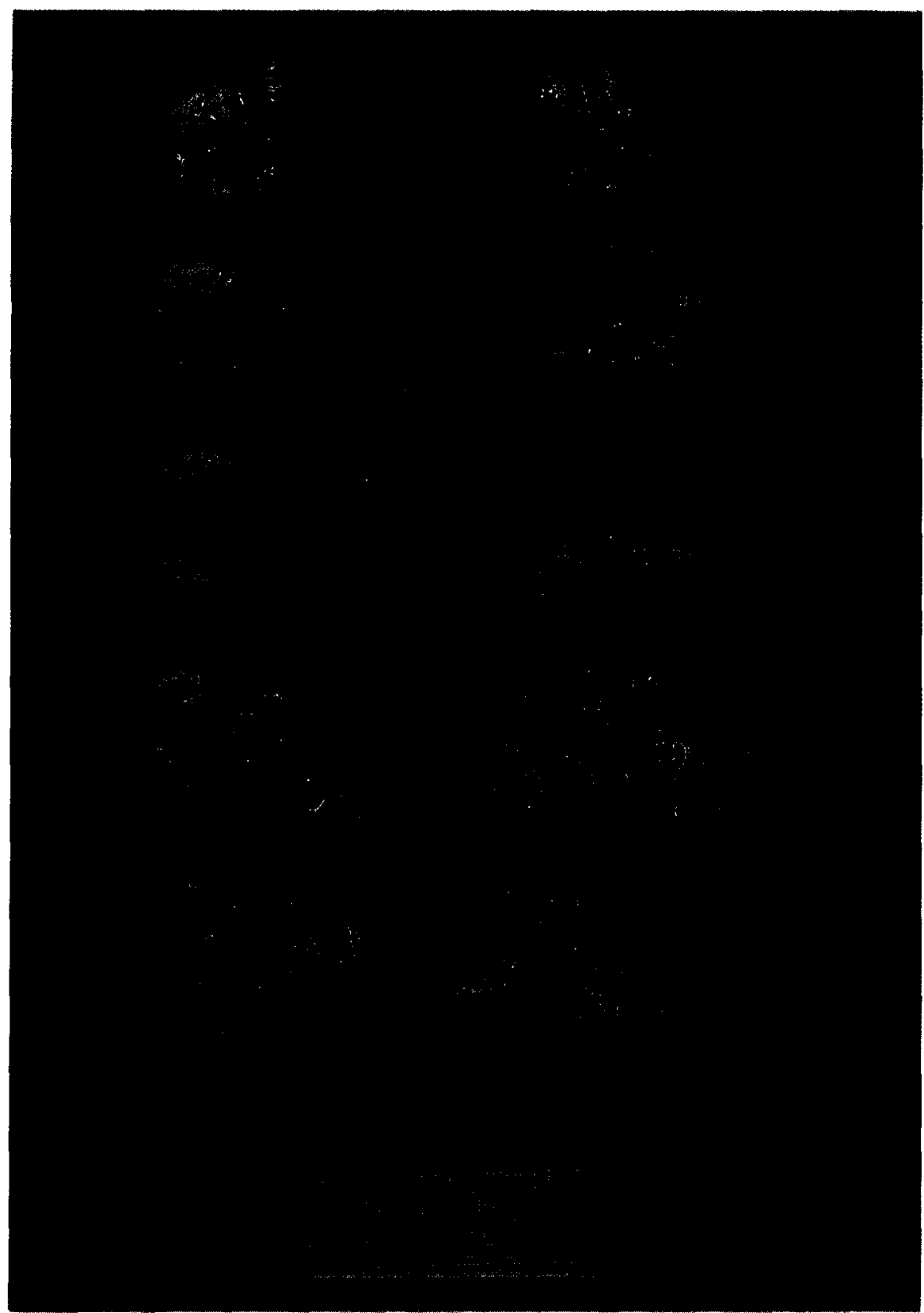

FIGURE 2. Panel I illustrates the difference between the TTC-exposed brains from a damaged rat (left) and a control rat (right) at $1 \times$ magnification. The white areas indicate regions of infarction. Slice A from panel I (damaged brain) is compared to brain slice B (respective slice from control brain) at sequentially higher magnifications to demonstrate the consistancy 


\section{Brain Slicing and Staining Procedure}

After neurological testing, the animals were sacrificed (decapitated), and the brains were quickly removed and placed into cold $\left(2-3^{\circ} \mathrm{C}\right) 0.9 \%$ saline solution to facilitate slicing. After $\mathbf{1 0} \mathrm{min}$, each brain was removed from the saline and placed on a cold glass plate. The olfactory bulb, cerebellum, and brain stem were removed and four cross-sectional cuts were made through the cerebrum with a hand-held razorblade. Cut I was made midway between the optic chiasm and the most anterior aspect of the frontal lobes. Cut II was made at the optic chiasm. Cut III was made at the infundibular stalk. Cut IV was made midway between the infundibular stalk and the caudal poles of the occipital lobes (Figure 1).

Each slice was placed in a petri dish and was completely covered with the staining solution consisting of $15 \mathrm{ml} \mathrm{4 \%} \mathrm{TTC}$ and $1 \mathrm{ml} 1 \mathrm{M}$ potassium phosphate (dibasic) diluted to $50 \mathrm{ml}$. The slices were incubated in the dark at $37^{\circ} \mathrm{C}$ for $30 \mathrm{~min}$ and turned once after $7.5 \mathrm{~min}$ to promote an even reaction. Normal tissue produces a brick red reaction product with the reduction of $\Pi \mathrm{C}$ by intracellular dehydrogenases, whereas ischemic tissue remains unreacted and uncolored (Figure 2, Panel I). Once exposed to $T T C$, the brain slices were preserved in $10 \%$ Formalin and were kept in the dark to preserve their color. Using the Levine rat and gerbil stroke models, we stained control (no carotid ligation), undamaged, and damaged (evaluated by neurological exam) animals to determine the applicability of the TC staining technique to cerebral tissue.

\section{Brain Infarct Sizing Technique}

The macroscopic images of stained brain slices were enlarged by projection onto a vertical screen using an opaque delineascope (Model 3252, American Optical Company). This increased both the accuracy and the reproducibility of the projected images obtained for the sizing technique. The anterior surface of each slice was designated " $a$," and the posterior surface was designated " $b$. ." For each side of each slice a tracing was made of the entire perimeter of the slice as well as the reacted and unreacted areas (Figure 1$)$. An area reference disc $\left(0.81 \mathrm{~cm}^{2}\right)$ was simultaneously projected and traced to record the degree of magnification for each projection (Figure 1). The unreacted area and total brain area of each slice were determined using a hand planimeter (Model 62000, Keuffel and Esser Company).

of macroscopic and histological determinations of infarct. Panel II compares slice $A$ (damaged) to slice $B$ (control) at $7 \times$ magnification. Areas of infarction can be identified in the right hippocampus and cortex of the damaged rat brain. The left side of the damaged brain and the control brain appear normal. The right hippocampi (areas in boxes) of the damaged (C) and undamaged (D) rat brains are shown at $200 \times$ magnification in Panel III. At $200 \times$ magnification almost the entire right hippocampus of the damaged rat $(C)$ is destroyed, whereas the control hippocampus (D) appears normal. The right $\mathrm{H} 1$ zones (areas in boxes) of the damaged (E) and undamaged (F) rat brains are shown at $400 \times$ magnification in Panel IV. The right $H 1$ zone of the damaged rat brain reveals necrotic neurons that exhibit extreme shrinkage, widened pericellular spaces, nuclear pyknosis, and cytoplasmic eosinophilia. The control H1 zone appears normal. 

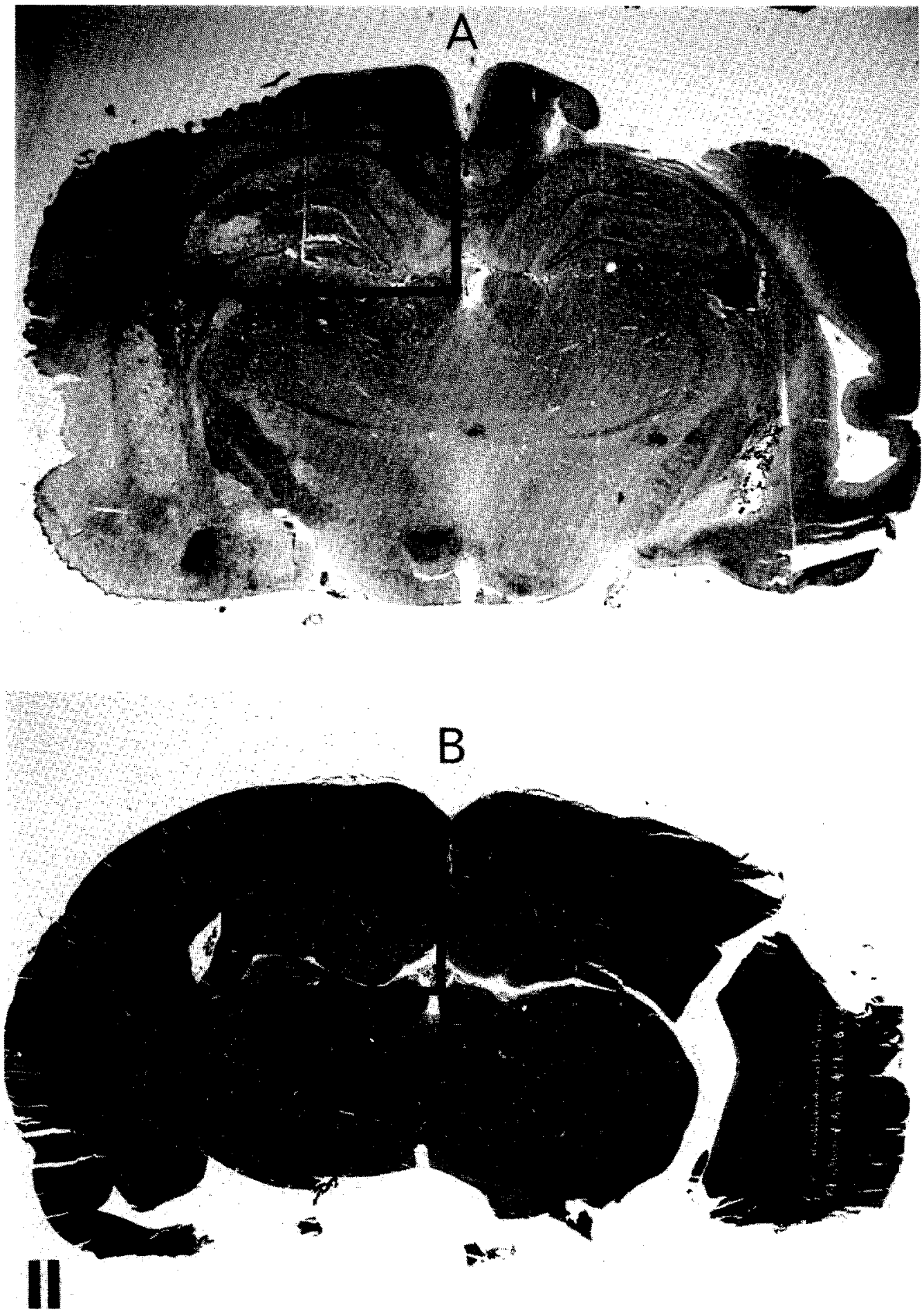

Figure 2. (cont.) 

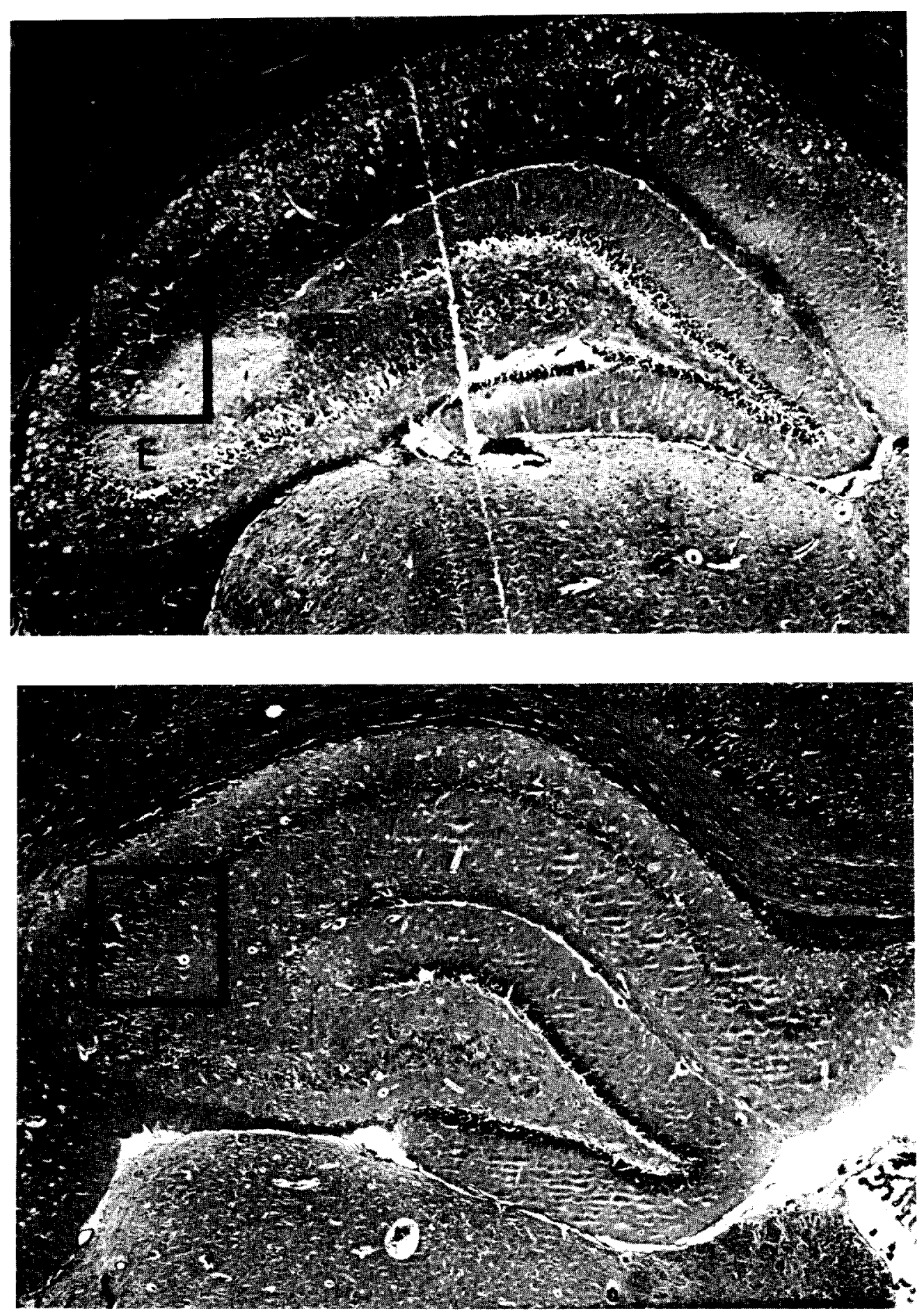

Figure 2. (cont.) 

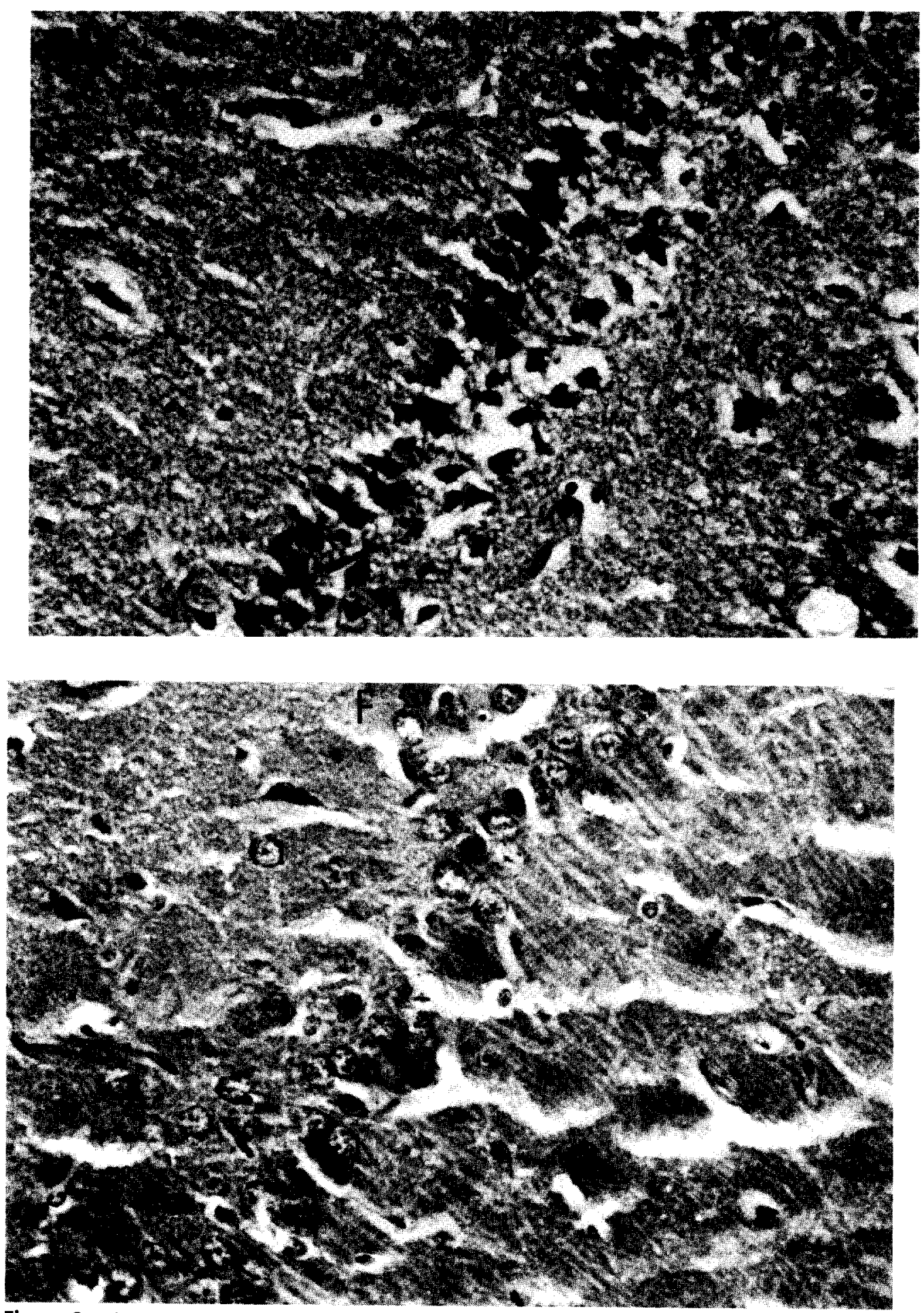

Figure 2. (cont.) 


\section{Histological Evaluation}

Brain slices stained with $\Pi T C$ were stored in Formalin until they were prepared for histological evaluation. Although desirable, in situ fixation would destroy the enzymes stained by TTC and hence could not be used. After macroscopic determination of infarct size, the fixed slices werc processed, embedded in paraffin, and cut in the usual manner. The sections were stained with (Harris's) hematoxylin and alcoholic eosin (H \& E) to distinguish gross infarct from normal tissue.

\section{RESULTS}

Conventional histological criteria verified that TTC selectively produces a red reaction product with noninfarcted cerebral tissue. In every case, the unreacted (and uncolored) tissue was ipsilateral to the carotid ligation and was consistent with histological identification of an infarct. From the $\Pi C$ stained slices we were able to determine the area of the infarct (Table 1) as a percentage of the total crosssectional area. The percentage of infarct correlated with the severity of the neurological impairment in both the rats and the gerbils.

Brains were obtained from seven Levine rats matched for age and weight. Rat No. 1 was the control (no carotid ligation and no hypoxic exposure), and its brain reacted uniformly with the TTC indicating no infarct (Figure 2, Table 1). Two rats underwent

TABLE 1 Area of Infarct as a Percentage of the Total Cross-sectional Area

\begin{tabular}{|c|c|c|c|}
\hline & $\begin{array}{c}\text { Total Cross-sectional } \\
\text { Area of } 10 \text { Faces } \\
\left(\mathrm{CM}^{2}\right)\end{array}$ & $\begin{array}{c}\text { TOTAL INFARCTED } \\
\text { CROSS-SECTIONAL } \\
\text { AREA OF } 10 \text { FACES } \\
\left(\mathrm{CM}^{2}\right)\end{array}$ & $\begin{array}{l}\text { PERCENTAGE OF } \\
\text { AREA INFARCTED }\end{array}$ \\
\hline \multicolumn{4}{|c|}{ Rat (control) } \\
\hline 1 & 11.6 & 0.0 & 0.0 \\
\hline \multicolumn{4}{|c|}{ Rat (neurologically intact) } \\
\hline 2 & 11.5 & 0.04 & 0.35 \\
\hline 3 & 12.4 & 0.03 & 0.24 \\
\hline \multicolumn{4}{|c|}{ Rat (neurologically impaired) } \\
\hline 4 & 13.4 & 5.2 & 39.0 \\
\hline 5 & 12.0 & 3.9 & 32.4 \\
\hline 6 & 11.5 & 5.1 & 44.3 \\
\hline 7 & 12.2 & 2.9 & 23.3 \\
\hline \multicolumn{4}{|c|}{ Gerbil (control) } \\
\hline 1 & $6.1^{a}$ & 0.0 & 0.0 \\
\hline \multicolumn{4}{|c|}{ Gerbil (neurologically intact) } \\
\hline 2 & $7.8^{a}$ & 0.0 & 0.0 \\
\hline 3 & $7.9^{a}$ & 0.0 & 0.0 \\
\hline \multicolumn{4}{|c|}{ Gerbil (neurologically impaired) } \\
\hline 4 & $5.4^{a}$ & 2.4 & 44.0 \\
\hline 5 & $6.4^{a}$ & 1.8 & 28.1 \\
\hline 6 & $4.7^{\mathrm{a}}$ & 1.0 & 21.3 \\
\hline
\end{tabular}

${ }^{a}$ Because of small size and tissue friability slice one was often not traced. Therefore only eight faces were used in the gerbil calculations. 
carotid ligation and hypoxic exposure but exhibited no neurological deficit. The brains of these rats remained unreacted in small areas suggesting limited cerebral damage (Table 1, animals 2 and 3). In the four neurologically impaired animals (Table 1 , rats $4-7$ ) large areas of infarct (average $=34.7 \%$ ) were identified macroscopically.

For each of the rat brain slices, the macroscopic identification of infarcted areas was compared with the histology of the same areas. The control brain, which reacted uniformly with $\mathrm{TTC}$, had no histologically identified areas of infarct. In the two behaviorally undamaged animals, only small areas of infarct were identified by the TTC stain (average $=0.3 \%$ ); the histological evaluation also revealed the same areas to be damaged. In the four neurologically impaired animals, large areas of infarction were identified histologically, and these areas corresponded to the identification of damaged tissue by TTC.

An individual example of TTC-stained brains from a damaged rat (left) and a control rat (right) is shown at $1 \times$ in Figure 2 (Panel 1$)$. Slice A (damaged brain) is compared to control brain slice $B$ (undamaged) at sequentially higher magnifications to illustrate the consistency of macroscopic and histological identification of infarct (Figure 2, II, III, and IV). At lower magnifications, it can be seen that there is extensive damage on the right side of the brain (ipsilateral to the carotid ligation) of the neurologically impaired rat. At higher magnifications (Panels III and IV), this brain revealed necrotic neurons with extreme shrinkage, widened pericellular spaces, nuclear pyknosis, and cytoplasmic eosinophilia. On the contralateral side, which reacted with $\Pi C$, there were only minimal histological changes consistent with reversible damage. Histologically demonstrable damage was most severe in the right hippocampus, neocortex, and corpus striatum as reported earlier for this model (Levine, 1960; Levy et al., 1975; Salford et al., 1973). At all magnifications the control brain appears normal.

A total of six gerbil brains were evaluated. The control brain (no carotid ligation) reacted uniformly with the TTC and was histologically normal. The brains of two gerbils that had undergone carotid ligation, but that had demonstrated no behavioral impairment, also reacted uniformly with the $\Pi T C$ and were histologically normal. The three gerbils that exhibited neurological impairment had large areas (average $=31 \%$ ) of the left hemisphere (ipsilateral to carotid ligation) that did not react with the TTC (Table 1). The histological evaluation of these three gerbil brains revealed that the infarct was restricted to the left hemisphere and correlated with the macroscopic determination of damage by the TTC. The hippocampus, neocortex, and corpus striatum of the ligated side were most severely damaged.

In both rats and gerbils, the percentage of infarct determined by the $\pi \mathrm{TC}$ correlated with the severity of the neurological deficit. The two neurologically impaired rats that were stained at $24 \mathrm{hr}$ (Table 1 , animals 4 and 5) had average 20-hr neurological scores of 3.7 and 3.4 , respectively (neurological score of $1.0=$ normal, 5.0 $=$ severe deficit). The percentage of the area of infarct was $39 \%$ for rat 4 and $32 \%$ for rat 5 . The two other neurologically impaired rats (Table 1 , animals 6,7 ) were stained at $48 \mathrm{hr}$. Rat 6 was more damaged and had an average neurological score of 3.9 and a $44.3 \%$ area of infarct. Rat 7 was less neurologically impaired than rat 6 with an average neurological score of only 2.8 , and the percentage of the area of 
infarct was $23.3 \%$. For the impaired gerbils, all of which were killed at $24 \mathrm{hr}$, the infarcted area averaged $31 \%$ for the three animals, and, again, the greater the impairment the larger the percentage of infarct.

To determine the inherent effects of the TTC on the histological appearance of the brain sections, two normal brains exposed to the TTC and two brains not exposed were sliced, fixed in Formalin, and stained with $\mathrm{H} \& \mathrm{E}$. No alteration in the $\mathrm{H} \& \mathrm{E}$ staining was detected in the TTC-stained tissues.

\section{DISCUSSION}

A method to quantify cerebral infarcts objectively would be a useful tool in the study of cerebral injury and potential protective agents. In this study we found that 2,3,5-triphenyltetrazolium chloride selectively reacts and produces a red product with noninfarcted cerebral tissue, whereas infarcted tissue remains unreacted and uncolored, thus permitting macroscopic determination of infarct location and size.

Since 1957 TTC has been used to identify and quantify myocardial infarcts (Boor and Reynolds, 1977; Cox et al., 1968; Fishbein et al., 1981; Jestadt and Sandritter, 1959; Lie et al., 1975; Nachlas and Shnitka, 1963; Sandritter and Jestadt, 1958). The selectivity of the TTC stain is based on its intracellular interaction with respiratory enzymes (dehydrogenases) (Jestadt and Sandritter, 1959; Nachlas and Shnitka, 1963; Sandritter and Jestadt, 1958). Metabolically active cells are rich in respiratory dehydrogenase activity, and the loss of such activity indicates irreversible damage to cells (Jennings and Kaltenbach, 1957; Wachstein and Meisel, 1955). As the dehydrogenases of viable tissue donate hydrogen ions to $\Pi T C$, it is reduced from a colorless compound to a red formazan-insoluble deposit. Irreversibly damaged tissue, devoid of respiratory enzyme activity, does not donate hydrogen ions, the TTC remains oxidized, and the tissue retains its unreacted color.

Although numerous studies have verified its utility, specificity, and reproducibility in the heart (Boor and Reynolds, 1977; Cox et al., 1968; Laga and Munro, 1972; Lie et al., 1975; Sandritter and Jestadt, 1958), Liszczak et al. (1984) found that TTC fails to identify cerebral infarcts under conditions of complete ischemia with no reflow. In the models we used, however, complete ischemia is not produced. In the gerbil stroke model, different levels of perfusion persist ipsilateral to the carotid ligation throughout the time course tested (Crockard et al., 1980; Ohno et al., 1984). In fact, several researchers have reported that cerebral ligation results in cerebral infarction in only $30-53 \%$ of the treated animals. The other $47-70 \%$ of the animals experience no neurological deficit (Levy and Brierley, 1978; McGraw, 1977; McGraw et al., 1976). In the Levine rat preparation, no detectable neurological impairment or histological evidence of damage is produced by carotid ligation alone. This suggests persistance of significant cerebral blood flow following ligation. To produce infarct in the Levine rat, hypoxia must follow carotid ligation. Our success with TTC indicated that this residual flow was sufficient to wash out the respiratory enzymes from the damaged cells and, hence, permit delineation of infarct by the red reaction product.

Fishbein et al. (1981) used linear regression to compare planimetry measurements of 54 gross slices of myocardium to microscopic giant histological sections and 
obtained a close correspondence of all areas of necrosis $(r=0.91)$. When sections with patchy necrosis were excluded, the correlation improved to $r=0.94$. These authors go on to stress that the loss of TTC staining ability (i.e., washout of dehydrogenase enzyme activity) occurs prior to demonstrable histological indexes of necrosis. That is, in the minutes or first few hours following an ischemic insult, light microscopy will tend to underestimate the extent of damage delineated by the loss of dehydrogenase reactivity. Ultrastructural studies by Fishbein et al. (1981) showed "complete agreement of the TTC technique and electron microscopy in identifying areas of necrosis." In the present study, brain staining was done at 24 and $48 \mathrm{hr}$ after the insult to minimize this early histological underestimation of damage by allowing time for tissue changes to occur. However, washout of cytosolic dehydrogenases was evidently complete at $24 \mathrm{hr}$, in that no further loss of staining was apparent in the animals staincd at $48 \mathrm{hr}$.

The observed histological changes were readily apparent and corresponded precisely to the areas delineated by the TTC stain. Areas that remained unreacted exhibited obvious histological signs of infarction. Likewise, areas that produced a red reaction product upon exposure to $\Pi \mathrm{TC}$ did not reveal histological signs of infarct. Histological techniques were used in this study only to confirm the stain's selectivity in the brain. Because in situ fixation compromises the TTC staining technique, we purposefully imposed severe insults and selected 24- and 48-hr end points to assure definitive histological changes indicative of infarcts. This approach avoided subtle changes in neuronal staining previously reported for early time points and after less severe insults (Brierley et al., 1973; Cammermeyer, 1973; Levy et al., 1975; Salford et al., 1973). The TTC technique, however, only delineates irreversibly damaged tissue.

Morphometric sizing of infarcts has proved an important means of objectively assessing myocardial infarcts and, hence, the results of proposed therapeutic interventions. From the brain slice tracings we were able to measure the percentage of the area infarcted. In this initial study we measured the infarcted versus noninfarcted cross-sectional areas of each of the ten brain slice faces. A more accurate indication of the infarct size could be obtained with computer-assisted planimetry and slice thickness measurements to convert these area measurements to approximate volumes.

The neurological score, used in this study and in previous work (Lundy et al., 1985) as an index of the degree of neurological impairment, appeared to correlate with the percentage of the area infarcted. Control and behaviorally unimpaired animals had very small or no infarcted areas. Neurologically impaired animals had obvious and significant infarcted areas, and, as the deficit became more severe, the infarcted area, expressed as percentage of the total cross-sectional area, also increased.

In summary, 2,3,5-triphenyltetrazolium chloride was shown to delineate noninfarcted versus infarcted cerebral tissue reliably in rat and gerbil brains. The stain's selectivity was verified by histological evaluation. Further, planimetry allowed quantification of brain infarct size, which correlated with functional assessment. We have found this technique to be an economical, simple, reproducible, and objective means of macroscopically quantifying cerebral infarct size. 
This study was supported in part by the Department of Surgery and the American Heart Association of Michigan.

Edward F. Lundy, M.D., Ph.D., was a Research Fellow of the American Heart Association of Michigan.

Our very special thanks to the members of the Ann Arbor Veterans Administration Hospital Medical Media Production Service, Robert McKnight, Dale Kennedy, and Daniel Cutler. Without their help and expertise, the illustrations for this manuscript could not have been completed. Our thanks to Stephanie Mitsos and Dr. Benedict Lucchesi for their help in applying the staining technique to cerebral tissue; to Mary Harper for her editorial help; and to Ilia Chernomordik and Andrew Cykiert for assistance with the gerbil and rat preparation.

\section{REFERENCES}

Boor PJ, Reynolds ES (1977) A simple planimetric method for determination of left ventricular mass and necrotic myocardial mass in postmortem hearts. Am / Clin Pathol 68:387-392.

Brierley JB, Meldrum BS, Brown AW (1973) The threshold and neuropathology of cerebral "anoxic-ischemic" cell change. Arch Neurol 29:367374.

Cammermeyer J (1973) "Ischemic neuronal disease" of Spielmeyer. Arch Neurol 29:391-393.

Cox JL, McLaughlin WW, Flowers NC, Horan LG (1968) The ischemic zone surrounding acute myocardial infarction. Its morphology as detected by dehydrogenase staining. Am Heart $J$ $76: 650-659$.

Crockard A, Fausto I, Hunstock AT, Smith RD, Harris RJ, Symon L (1980) Cerebral blood flow and edema following carotid occlusion in the gerbil. Stroke 11:494-498.

Fishbein MC, Meerbaum S, Rit J, Lando U, Kanmatsuse K, Mercier JC, Corday E, Ganz W (1981) Early phase acute myocardial infarct size quantification: Validation of the triphenyl tetrazolium chloride tissue enzyme staining technique. Am Heart / 101:593-600.

Harrison MJG, Brownbill D, Lewis PD, Russell RWR (1973) Cerebral edema following carotid artery ligation in the gerbil. Arch Neurol 28:389-391.

Jennings RB, Kaltenbach JP (1957) Enzymatic changes in acute myocardial ischemic injury. Arch Pathol 64:10.

Jestadt R, Sandritter HW (1959) Erfahrungen mit der TTC (triphenyltetrazoliumchlorid) reaktion fur die pathologisch-anatomische diagnose des frischen herzinfarktes. Kreislaufforsch 48:802.

Kahn K (1972) The natural course of experimental cerebral infarction in the gerbil. Neurology 22:510-515.

Laga EM, Munro HN (1972) Computer-assisted quantitative videoscanning technique for measuring cross-sectional areas and dimensions of tissue components. Med Biol Eng 10:473-482.

Levine S (1960) Anoxic ischemic encephalopathy in rats. Am / Pathol 36:1-17.

Levine S, Sohn D (1969) Cerebral ischemia in infant and adult gerbils. Arch Pathol 87:315-317.

L evy DE, Brierley JB (1978) Delayed pentobarbitol administration limits ischemic brain damage in gerbils. Ann Neurol 5:59-64.

Levy DE, Brierley JB, Silverman DG (1975) Brief hypoxia-ischemia initially damages cerebral neurons. Arch Neurol 32:450-456.

Lie JT, Pairolero PC, Holley KE, Titus IL (1975) Macroscopic enzyme-mapping verification of large, homogeneous, experimental myocardial infarcts of predictable size and location in dogs. I Thorac Cardiovasc Surg 69:599-605.

Liszczak TM, Hedley-Whyte ET, Adams JF, Han DH, Kolluri VS, Vacanti FX, Heros RC, Zervas NT (1984) Limitations of tetrazolium salts in delineating infarcled brain. Acta Neuropathol 65:150157.

Lundy EF, Dykstra J, Luyckx BA, Zelenock CB, D'Alecy LG (1985) Reduction of neurologic deficit by 1,3-butanediol induced ketosis in Levine rats. Stroke 16:855-860.

Lundy EF, Luyckx BA, Zelenock GB, D'Alecy LG (1984) Butanediol induced cerebral protection from ischemic-hypoxia in the instrumented Levine rat. Stroke 15:547-552.

McGraw CP (1977) Experimental cerebral infarction effects of pentobarbital in Mongolian gerbils. Arch Neurol 34:334-336.

McGraw CP, Pashayan AG, Wendel OT (1976) Cerebral infarction in the mongolian gerbil exacer- 


\section{E. F. Lundy et al.}

bated by phenoxybenzamine treatment. Stroke 7:485-488.

Nachlas MM, Shnitka TK (1963) Macroscopic identification of early myocardial infarcts by alterations in dehydrogenase activity. Am / Pathol 42:379-396.

Ohno K, Ito U, Inaba $Y$ (1984) Regional cerebral blood flow and stroke index after left carotid ar. tery ligation in the conscious gerbil. Brain Res 297:151-157.

Payan $\mathrm{HM}_{z}$ Conrad IR (1977) Carotid ligation in gerbils. Stroke 8:194-196.
Salford LG, Plum F, Brierley JB (1973) Graded hypoxia-oligemia in rat brain. Arch Neurol 29:234238.

Sandritter HW, Jestadt R (1958) Triphenyltetrazoliumchlorid (TTC) als reduktionsindikator zur of makroskopischen diagnose des frischen herzinfarktes. Verh Dtsch Ges Pathol 41:165.

Wachstein M, Meisel E (1955) Succinic dehydrogenase activity in myocardial necrosis. Am / Pathol 31:353. 